\title{
Short-Term Load Forecasting Based on RS-ART
}

\author{
Tao Yang, Feng Zhang, Qingji Li, and Ping Yang \\ College of Information and Electrical Engineering, Shenyang Agriculture University, \\ Shenyang 110061, China
}

\begin{abstract}
This paper presents a short-term electric load forecasting method based on Autoregressive Tree Algorithm and Rough Set Theory. Firstly, Rough Set Theory was used to reduce the testing properties of Autoregressive Tree. It can optimize the Autoregressive Tree Algorithm. Then, Autoregressive Tree Model of Short-term electric load forecasting is set up. Using Rough Set Theory, the attributes will be reduced off; whose dependence is zero, through knowledge reduction method. It not only avoids the complexity and long training time of the model, but also considers various factors comprehensively. At the same time, this algorithm has improved the prediction rate greatly by using automatic Data Mining Algorithms. Practical examples show that it can improve the load forecast accuracy effectively, and reduce the prediction time.
\end{abstract}

Keywords: Rough Set (RS), Autoregressive Tree Algorithm (ART), Short-Term Electric Load Forecasting, Data Mining.

\section{Introduction}

Short-Term Load Forecasting is a system load forecasting before one day or one week, and an important work of electricity production department. It has great significance in power system operation and design. Power system security and economic operation are based on Short-Term Load Forecasting. Load forecasting has very close relations with generation companies, power companies and transmission companies under electricity market. For generation companies, load forecasting can make plans, maintenance plans and offer a basis of quoted price. For power companies, load forecasting can provide the basis to develop power purchase plan for the power suppliers. For transmission companies, load forecasting is the basis to develop plans and keep safe, reliable, economic operation. Load forecasting plays an important role in electricity market decision support systems.

Short-Term Load Forecasting has many traditional prediction algorithms such as single regression method, multiple linear regression and Fourier Component Method, etc. Although these algorithms are mature and simple, these algorithms are linear model methods. When they meet non-linear problem, they appear problem. This article proposes Autoregressive Tree Algorithm based on Rough Set Theory (the RS-ART Algorithm), which can overcome the shortcomings of traditional algorithm. ART algorithm uses the piecewise linear regression mode, which can meet non-linear problem effectively. The Rough Set Algorithm can reduce the attribute of Autoregressive Tree. And it can increase the forecasting precision and reduce forecasting time. 
Using RS-ART Algorithm not only can meet non-linear problem effectively, but also can enhance forecasting precision and reduce forecasting time.

\section{Short-Term Forecasting Model Based on RS-ART Algorithm}

\subsection{Autoregressive Tree (ART)}

The most common type of model used for time-series analysis is the linear autoregressive (AR) model. A linear autoregressive model of length $p$, denoted $A R(p)$, is described by Equation 1:

$$
f\left(y_{t} / y_{t-p}, \cdots, y_{t-1}, \theta\right)=\prod_{i=1}^{L}\left(m_{i}+\sum_{j=1}^{P} b_{i j} y_{t-j}, \sigma_{i}^{2}\right) \phi_{i}
$$

Where $N\left(\mu, \sigma^{2}\right)$ is a normal distribution with mean $\mu$ and variance $\sigma$, and $\theta=\left(m, b_{1} \ldots\right.$ $\left.b_{p}, \sigma 2\right)$ are the model parameters.

An Autoregressive Tree (ART) Model is a piecewise linear autoregressive model in which the boundaries are defined by a decision tree, and the leaves of the decision tree contain linear autoregressive models. An ART $(p)$ model is an ART model in which each leaf of the decision tree contains an $A R(p)$ model, and the split variables for the decision tree are chosen from among the previous $p$ variables in the time series. Supposes each leaf node is $l_{i}$ and related target function is $\varphi_{i}$, then $(A R T(p))$ model can be described by Equation 2:

$$
f\left(y_{t} / y_{t-p}, \ldots, \theta\right)=\prod_{i=1}^{L} N\left(m_{i}+\sum_{j=1}^{P} b_{i j} y_{t-j}, \sigma_{i}^{2}\right) \phi_{i}
$$

\subsection{Rough Set (RS)}

Rough Set Theory is one kind of data analysis theory which is raised in 1982 by Professor Z.Pawlak in University of Technology in Poland. It is a kind of new mathematical instrument that processes fuzzy and the uncertain knowledge. Its main idea is that classifying is invariable under premise. It derives the decision or the classifying rule of the question by knowledge reduction. The smallest condition attributes subset which is obtained by attribute reduction may replace the original policy-making table condition attribute set, and it does not affect the classifying ability of the policymaking table. The application of Rough Set in the data mining can enhance the analysis and study ability of incomplete data in huge database. And it has widespread application prospect and the use value.

\subsection{RS-ART}

This method contains two processes: one uses RS to do attribute reduction and construct a new study and test sample, another one uses ART to complete the duty of forecasting. Firstly historical data is used, which contains all characteristic parameters to create decision-making table $\mathrm{S}$, its condition attribute set is $\mathrm{C}$ and policy-making attribute is D. Secondly all attributes are separated, which are in the policy-making 
table to standardize expression way, dependence of all conditional attributes are calculated in the current environment. Thirdly conditional attributes are removed, whose dependence is 0 , a new study sample set of ART obtained. Fourthly ART is studied and trained again, test input data, which is reformed and combined from reduced attribute set $\mathrm{C}_{1}$ and corresponding primary data to complete forecasting task. RS-ART to forecast is as follows:

At the beginning, the $\mathrm{n}$ study sample is determined, which only contains reduced attribute set $\mathrm{C}_{1}$. Then reduced data is preprocessed. Bayes technology is used to train model data. Finally, ART algorithm is used to carry on the forecasting.

\section{The Algorithm of Short-Term Forecasting Based on RS-ART}

\subsection{Attribute Reduction Based on RS}

According to historical data, decision-making table is created to decide attribute value $\mathrm{S}=<\mathrm{U}, \mathrm{C} \cup \mathrm{D}>$, which $\mathrm{C}$ is weather information (including wind speed $\mathrm{w}$, temperature $\mathrm{t}$, humidity $\mathrm{h}$, weather condition s and barometric pressure $\mathrm{p}$ and so on) and $\mathrm{D}$ is the load value in forecasting day, the step reduced the attributes is as follows:

\section{(1) Discrete All Attribute In Policy-Making Table}

As we known, Temperature range is $[-1,20]$ in October in Shenyang. The range is divided into 6 parts. $[-1,0]$ is marked $0,[0,4]$ is marked $1 \ldots[16,20]$ is marked 5 . Wind speed and humidity are the same as temperature. Weather condition is divided (including fine, cloudy, rainy day) into 0,1 , and 2 . That barometric pressure is divided with the method is the same as the above. Load data from high to low can be divided into 2,1 , and 0 .

(2) Calculate Dependence of All Conditional Attributes

$\mathrm{D}$ to $\mathrm{C}$ dependence is as follows:

$$
r_{c}(D)=\frac{\operatorname{card}(\operatorname{POS}(D))}{\operatorname{card}(U)}=1
$$

It shows that policy-making table is completely coordinated. To $\mathrm{W}$ is:

$$
r_{c}(D)-r_{c-\{W\}}(D)=0
$$

Likewise, calculate the rest conditional attributes.

(3) Remove Attributes Dependence is 0

Remove wind speed, humidity and barometric pressure etc., whose dependence is 0 .

(4) Obtain Final Attribute Reduction Set

Through the attribute reduction, final attribute reduction set $\mathrm{C}_{1}$ \{temperature, weather condition \} is obtained. 


\subsection{Preprocess Reduced Data}

During gathering process of load forecasting historical data in electrical power system, because of various kinds of reason, there are many unusual data such as erroneous data, flaw data and so on. The identification and revision of these unusual data is the foundation of forecasting model to do perfect forecasting.

According to the type of unusual data, the different processing method is used in this article. The steps to process these unusual data are as follows:

\section{(1) Erroneous Data Processing}

The erroneous data is filtered through assigning value scope of these data. For example, set temperature value scope, $-1{ }^{\circ} \mathrm{C} \sim 20^{\circ} \mathrm{C}$.

(2) Flaw Data Processing

Flaw data is generated from primary data and filtered data. When the time span of the flaw data is not too large, Linear Interpolation Method can be used to process. For example, data $\mathrm{n}, \mathrm{n}+1, \mathrm{n}+\mathrm{j}$ are as known as:

$$
T_{n+j}=T_{n}+\frac{T_{n+j}-T_{n}}{i}, 0<j<i
$$

When the time span of the flaw data is large, the next day data can be used to fill.

(3) Incompatible Data Processing

Existing load data is 1 each minute, but meteorological data is 6 each hour. Therefore, transforming the data density is needed. All data become 4 each hour. The concrete plan is that 4 load data are extracted each hour and meteorological data are used by Linear Interpolation Method to fill.

\subsection{Load Forecasting Based on ART Algorithm}

The task of forecasting is calculation of the distributions of decision tree for future observations in the sequence. The posterior distribution for the variable $Y_{T+1}$ is a function of a single leaf node in the tree. Using the conjugate priors, each leaf in the tree has a conditional $\mathrm{t}$-distribution for this variable. Using the normal distribution $f_{t}\left(y_{t} / y_{t}\right.$ $p \ldots y t-1, \theta)$ evaluated at the value of $\theta_{i}$, the data are most likely given as the maximum a posteriori (MAP) value:

$$
\tilde{\theta}_{i}=\operatorname{argmax} \prod_{x^{t} a t l^{i}} f\left(x^{t}{ }_{p+1} / x_{1}{ }^{t}, \cdots, x_{p}{ }^{t}, \theta, s\right) P\left(\theta_{i} / s\right)
$$

Then $\mathrm{t}$ - distribution is gotten to make the final forecasting. Using this formula iteratively, whether forecast is more than one data in the future.

\section{Case Study}

24 hour integral point load data and meteorological data are used in October, 2007 in Dongling, Shenyang. The first $2 / 3$ data is used to train, and the rest to test and verify. 


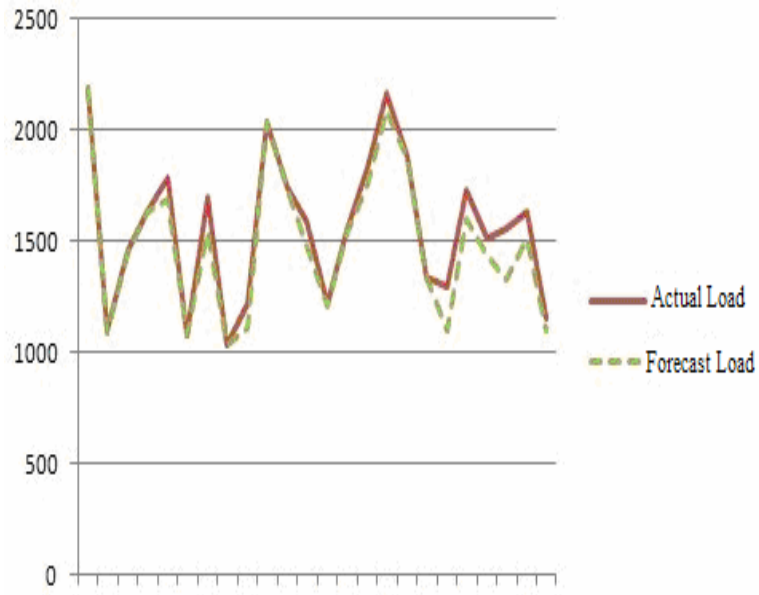

$\begin{array}{llllllllllll}1 & 3 & 5 & 7 & 9 & 11 & 13 & 15 & 17 & 19 & 21 & 23\end{array}$

Fig. 1. Forecasts Curve and Measured Curve

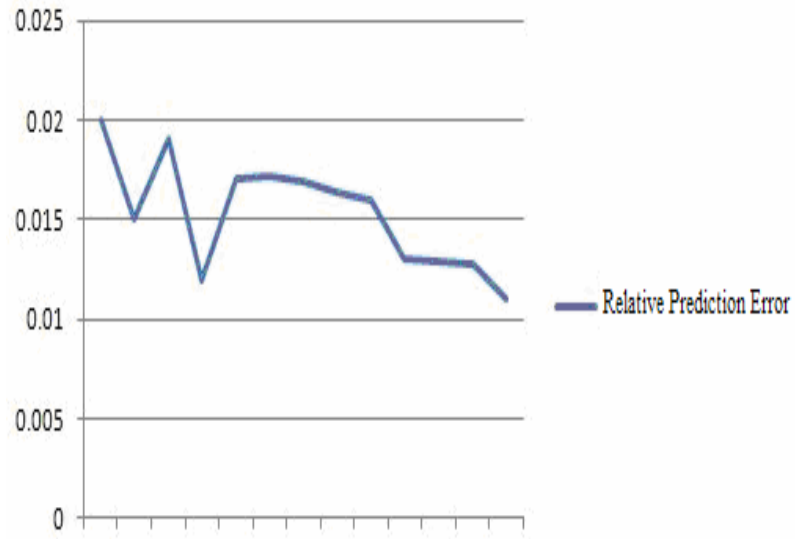

$\begin{array}{llllllllllllll}1 & 2 & 3 & 4 & 5 & 6 & 7 & 8 & 9 & 10 & 11 & 12 & 13\end{array}$

Fig. 2. The Relative Prediction Error Curve

The RS-ART model above is trained to achieve the certain forecasting precision without fitting too much. Use well trained model to forecast 24 hour load in October 15, 2007 to get corresponding forecasting result. Figure 1 is Forecasts Curve and Measured Curve figure, in which solid line is Actual Load $Y$ Curve and dashed line is Forecast Load $Y_{d}$ Curve. Figure 2 is Relative Prediction Error Curve.

As the forecasting result indicates, the relative error and the average error of RS-ART Algorithm are $2 \%$ and $1.89 \%$. 


\section{Conclusion}

This article proposes an ART Algorithm based on Rough Set. This algorithm has all advantage of ART Algorithm. And Rough Set can be used to do the attribute reduction completely. Practical examples show that it can improve the load forecast accuracy effectively, and reduce the prediction time.

\section{References}

1. Wang, J.-m., Ren, R.-y.: Application of dummy variables in electric consumption forecasting. Power Demand Side Management 9(3), 16-19 (2007)

2. Li, L., Xia, D., Yang, Z.: Short-Term Load Forecasting Using Artifical Neural Network. In: Proceedings of The Csu-Epsa, vol. 6(3), pp. 34-41 (1994)

3. Yu, X., Niu, C., Li, J.: Electric load forecast based on decision tree and expert system. Journal of North China Electric Power University 32(5), 58-61 (2005)

4. Zhu, T.-y., Zhang, X.-z., Li, Y.-q., Zhang, Y., He, C.-y.: A New Algorithm of Advancing Weather Adaptability Based on Arima Model for Day-ahead Power Load Forecasting. In: Proceedings of the Chinese Society For Electrical Engineering, vol. 26(23), pp. 14-18 (2006)

5. Shen, X., Wu, Y., Xiong, X.: Short-term Load Forecasting Based on BMPMR. In: Proceedings of The Csu-Epsa, vol. 19(4), pp. 45-48 (2007)

6. Thissen, U., Van Brakel, R., de Weijer, A. P.: Using support vector machines for time series prediction. Chemometrics and Intelligent Laboratory Systems 69(2), 35-49 (2003) 